\title{
Order and disorder in intellectual history. Spinoza's case in the light of complexity
}

\author{
Maxime Rovere ${ }^{1}$ \\ ${ }^{1}$ IRHIM, ENS Lyon, 15 parvis Descartes, 69342, Lyon, France
}

\begin{abstract}
This article presents the conception of order and disorder developed by the philosopher B. Spinoza (1632-1677), and shows how this theory supports the intelligibility of nature without attributing any kind of organization to nature. From these principles, the author questions the use of causality in intellectual history, and sketches the possibility of a complex history based upon the disorder of interactions.
\end{abstract}




\title{
Ordre et désordre en philosophie : le cas Spinoza au défi de la complexité.
}

\author{
Maxime Rovere ${ }^{1}$ \\ ${ }^{1}$ Chercheur associé, IRHIM, ENS de Lyon, 15 parvis Descartes, 69342, Lyon.
}

\begin{abstract}
Cet article présente la conception de l'ordre et du désordre développée par le philosophe Spinoza $(1632$ - 1677), et montre comment cette conception défend l'intelligibilité de la nature sans lui attribuer une organisation. L'auteur en tire parti pour interroger l'usage de la causalité en histoire des idées, et esquisser la possibilité d'une histoire complexe, fondée sur le désordre des interactions.
\end{abstract}

\section{Introduction}

Comment une approche inspirée des théories du chaos et de la complexité peut-elle éclairer l'histoire des idées ? Pour poser la question autrement, comment l'histoire de la philosophie, ou l'histoire intellectuelle, peuvent-elles exploiter la notion de désordre pour rendre compte des éléments qui ont donné naissance à une philosophie particulière?

La plupart des historiens, animés par un souci de clarté, tâchent de mettre de l'ordre parmi les facteurs qui favorisent l'émergence de certaines innovations intellectuelles, et les ayant ordonnés et réunis, estiment être allés au bout de ce qu'une science historique peut faire. Pourtant, il est possible de défendre en ce domaine l'intelligibilité plus poussée de ces phénomènes émergents, à condition de mettre au point une épistémologie de l'histoire susceptible de rendre justice au désordre.

Pour aborder ces problèmes et développer une conceptualité adaptée, le cas du philosophe B. de Spinoza (1632 - 1677) est particulièrement intéressant. Spinoza dispose sur cette question d'une théorie remarquable : il soutient qu'il n'y a ni ordre ni désordre dans la nature. Le présent article se propose donc, dans un premier temps, d'étudier la conception propre à Spinoza de ces deux notions, avant de la retourner sur elle-même afin de formuler, dans un deuxième temps, de nouvelles directions en histoire intellectuelle.

\section{Ordre et désordre, entre imagination et infini.}

Dans une lettre adressée de Voorbug le 20 novembre 1665 à Henry Oldenburg, secrétaire de la Royal Society à Londres, Spinoza écrit : «Je voudrais avertir en premier lieu que je n'attribue à la nature ni beauté ni laideur, ni ordre ni confusion. Car les choses ne peuvent être dites belles ou laides, ordonnées ou confuses, que pour notre imagination ${ }^{\mathrm{i}}$. » Comme on voit, le contraire de l'ordre ici n'est pas le désordre, mais la confusion, et cette alternative est présentée en parallèle avec l'opposition entre beauté et laideur, ce qui la rapproche de considérations esthétiques qui ramènent l'ensemble du côté de la composition du corps humain. Spinoza considère, en l'occurrence, que le concept d'ordre n'exprime rien d'autre qu'une certaine commodité d'imagination. Ce qui est ordonné est ce qu'il nous est le plus facile de nous représenter par l'imagination, qui dépend elle-même de nos sens. Comme chacun sait, une salle dont les chaises sont disposées en rangs linéaires est plus facile à imaginer qu'une salle où elles sont disposées de manière aléatoire. Sur ces fondements, Spinoza entend donc retirer à la distinction entre ordre et confusion toute pertinence pour décrire la nature. Dans un autre texte (l'appendice à la première partie de l'Ethique), c'est bien l'alternative entre ordre et "désordre » qui est récusée $e^{\mathrm{ii}}$. Mais que reste-t-il à prédiquer de la nature, et que reste-til de son intelligibilité, lorsqu'on y efface toute frontière entre ordre et désordre ? Perplexe, Oldenburg répond 
ceci : « Je n'arrive pas assez bien à comprendre comment nous pouvons exclure de la nature l'ordre et la symétrie, comme vous semblez le faire, d'autant que vous reconnaissez vous-même que tous les corps sont entourés par d'autres, et qu'ils se déterminent les uns les autres, selon un rapport précis et constant, à exister et à opérer toujours au service d'un même rapport de mouvement et de repos chez tous à la fois, ce qui est très précisément, semble-t-il, la raison formelle d'un véritable ordre ${ }^{\mathrm{iii}}$. ’

La difficulté dont témoigne Oldenburg est que si l'on conçoit la nature comme intelligible, et que cette intelligibilité se conçoit par mouvement et repos (les deux concepts majeurs de la physique mécaniste, dont les deux hommes se revendiquent), on ne voit pas pourquoi l'articulation des mouvements et des repos en une totalité invariable ne correspondrait pas à la notion d'ordre. A cet étonnement, Spinoza ne répondra pas directement, mais on peut reconstituer une réplique possible à partir de sa métaphysique. Elle consiste à prendre en compte la complexité infinie de la nature - car c'est autour de cet infini que toute sa conception métaphysique s'articule. En effet, l'infini considéré en soi est pour Spinoza, comme pour tous les cartésiens, un concept purement positif, et donc parfaitement clair à l'entendement humain, car il est absolument simple, indivisible et unique ${ }^{\mathrm{iv}}$. Or, une essence infinie simple ne peut pas être contraire à ellemême ; par conséquent, la simplicité de l'être infini nous garantit que la nature n'est pas un désordre (dès lors que le concept de désordre enveloppe, lui, conflits et contradictions).

Mais il existe aussi une autre figure de l'infini, que Spinoza préfère appeler indéfini ${ }^{\mathrm{v}}$, susceptible d'une analyse qui en isole les parties. Pour décrire cette figure particulière de l'infini, Spinoza considère la possibilité de diviser et diviser encore les parties de l'espace ${ }^{\text {vi }}$. Ce raisonnement fonctionne aussi dans l'autre sens, lorsque l'on envisage de compliquer sans cesse les rapports entre les choses, qui en s'associant deviennent de plus en plus sophistiquées $^{\text {vii }}$. Or, en poursuivant la sophistication de ces rapports ad libitum, on doit admettre qu'on passe un seuil au-delà duquel la technique de l'analyse, qui consiste à distinguer les éléments un à un pour les penser clairement, ne fonctionne plus: la logique globale du système de la nature échappe à une conception analytique fondée sur ses éléments.

On pourrait donc parler d'un hiatus entre un infini ontologique (l'infinité d'essence de la nature, qui est toute simple) et un infini épistémologique (qui laisse nos capacités intellectives se noyer dans les complications). Mais Spinoza n'admet pas aussi facilement la limitation de nos capacités intellectuelles. Il semble donc plus correct de parler d'une double intelligibilité, barrée par un hiatus : dans l'ensemble, l'idée simple et indubitable que nous avons de l'infini nous assure qu'il n'y a pas de désordre dans la nature; dans le détail, nous pouvons saisir des lois et des relations qui nous confirment que nous pouvons y observer un certain ordre; pourtant, entre la nature du Tout et les lois particulières, nous ne savons pas démontrer le lien (qui ne peut être qu'un objet d'intuition) ; ce qui montre que l'impression d'ordre que nous avons est plus imaginaire que rationnelle.
Ainsi, lorsque l'on tient compte de l'infinité des rapports qui définissent la nature et de l'impossibilité de donner une place et une fonction à des éléments considérés séparément, on peut dire qu'il n'y a pas d'ordre dans la nature, puisqu'il est structurellement impossible d'établir un rapport clair entre une chose singulière et le Tout. En d'autres termes, un ensemble infini est trop grand pour y localiser quoi que ce soit. Pourtant, ce système infini de choses n'est pas non plus un désordre, puisque des relations locales se laissent néanmoins décrire entre des éléments qui se laissent isoler : nous faisons suffisamment l'épreuve de formes d'intelligibilité locales pour admettre qu'il n'y a pas non plus de désordre dans la nature.

On pourrait donc résumer la métaphysique de Spinoza de la manière suivante : l'univers est le fait d'un être simple et infini, capable de se déployer en une infinité de variations infinies; et bien que la nature entière se ramène à une seule «substance », l'unicité de cette entité ultime n'est qu'un abus de langage. Elle est unique au sens où il n'y en a pas d'autre, mais il est inexact de dire qu'elle est une, puisqu'elle est infiniment multiple.

En somme, la philosophie de Spinoza, animée par un immense effort pour penser par delà l'alternative entre ordre et désordre, isole d'un côté un rapport imaginaire au monde qui dépend seulement des capacités de notre imagination; lorsque l'on considère les choses selon ce mode, on considère comme ordonné ce qui est facile à imaginer, et comme désordonné ce qui est difficile ; de ce point de vue, ordre et désordre désignent le plus ou moins de confusion engendré dans l'esprit du fait des limites de l'imagination, c'est-à-dire du corps humain. Mais d'un autre côté, l'intelligibilité de la nature est maintenue pardelà l'ordre et le désordre, en tant qu'elle est l'expression d'un être infini que nous connaissons très clairement soit dans sa simplicité première, soit dans ses expressions localement intelligibles. Sa cohérence est garantie par la simplicité de l'idée d'infini (en ce sens, la nature n'est pas désordonnée), mais pour saisir et comprendre cette cohérence, il faudrait embrasser l'infinité numérique des choses de la nature, ce qui est impossible (en ce sens, la nature n'est pas ordonnée). La grande puissance de cette pensée est qu'elle ne suppose aucun ordre dans la nature, bien qu'elle en affirme l'intelligibilité. En définitive, l'intelligibilité peut se passer d'un ordre ontologique, sans pour autant être une construction de l'esprit.

\section{Le brouhaha de l'histoire.}

Jusqu'à présent, l'abordage purement conceptuel de la pensée de Spinoza m'a conduit à la livrer comme une proposition littéralement anhistorique. Comment cette conception peut-elle être retournée sur elle-même, afin de nous guider dans la manière dont nous faisons de l'histoire intellectuelle ? Observons d'abord que tout au long de cette présentation, l'intervention du commentateur s'est voulue entièrement transparente. Quelles sont les déformations que j'ai imprimées à la pensée de Spinoza, même sans le vouloir ni le savoir ? Et si (par impossible) il n'y en avait aucune, le sens de ces propos peut-il être le même au XXIe siècle qu'au XVIIe ? Evidemment non. Dans la manière anhistorique de faire 
de l'histoire de la philosophie, il y a donc un impensé flagrant. Pour le surmonter, il vaut la peine de se poser des questions historiennes, et c'est ici que la complexité va surgir dans sa forme contemporaine.

En effet, face à un texte philosophique quelconque, les historiens ont pour habitude de mobiliser les traditions qui préexistent au philosophe, les lectures qu'il ou elle a faites, les polémiques de son temps, et c'est ainsi qu'ils vont chercher à dégager ce qui permet de percevoir son originalité. Mais on observe alors une sorte de point d'arrêt : la grande finesse dont certains font preuve dans l'étude du contexte et des antécédents ne s'articule pas exactement au surgissement de l'idée originale. Le rapport intelligible qui devrait exister entre les facteurs contextuels et la naissance de l'œuvre se refuse : on admet bientôt une part d'indiscernable, d'inexplicable, qui serait liée au "génie» de la personne et au mystère du « singulier » qui échapperait à l'historien ${ }^{\text {viii }}$.

Dans le cas de Spinoza, on doit admettre que l'extraordinaire effervescence intellectuelle à Amsterdam au XVIIe siècle a de quoi dérouter. Ce moment chaotique ne se laisse appréhender qu'avec des outils capables de rendre justice à tout autre chose qu'à des causalités linéaires et aux mystères du singulier. Brossons à grands traits cette situation, afin de rendre perceptible l'analogie structurelle avec l'excès de complication que Spinoza a repéré dans les choses de la nature. Pendant que les guerres de religion entre catholiques et protestants viennent de déchirer l'Europe (surtout la France), la Hollande du XVIIe s., en pointe dans un commerce maritime qui s'étend désormais de la Chine au Brésil, est l'Etat le plus riche des Provinces-Unies, qui ont obtenu leur indépendance de 1'Espagne après 80 ans de guerre (traité d'Utrecht, 1638). Pendant cette guerre, catholiques et calvinistes ont su faire taire leurs différends; par conséquent, les Pays-Bas s'affirment comme une République de régents où l'Etat tient à distance les velléités de contrôle de l'Eglise calviniste, majoritaire. Cette tolérance favorise la multiplication plus ou moins pacifique de mouvements religieux qui se divisent sur tout ce qui touche à la religion chrétienne : le texte de la Bible et ses traductions, les interprétations, le statut de la vérité du message divin, les institutions, etc. En 1628, le synode de Dordrecht valide une traduction d'Etat (Statenvertaling) qui ne fait qu'alimenter les controverses. Dans le même temps, cette situation attire les savants et les étudiants étrangers qui, après le français René Descartes (1596 - 1650), viennent étudier la philosophie naturelle. Le cartésianisme a l'avantage de simplifier les catégories scolastiques en les remplaçant par une conceptualité plus simple ; néanmoins, les néothomistes continuent d'occuper des postes-clés; et entre ces deux camps, un éventail de propositions hybrides développent la «philosophia novantica» (à la fois ancienne et moderne).

En somme, on pourrait dire qu'une très grande liberté laisse s'épanouir un nombre d'options variées qui alimentent des controverses, tandis qu'un changement de regard permet de multiplier les découvertes qui rendent les domaines du savoir plus autonomes. Dans ce contexte, les philosophes évoluent dans une sorte de brouhaha de discours, de manuscrits et de publications où il est difficile de déterminer les rôles des uns et des autres.

\section{L'effort historien de "clarté".}

Pour sortir du bruit où tout le monde parle en même temps, une méthode consiste à établir des lignes de démarcation. Dans un livre publié en 2001 sous le titre Radical Enlightenment. Philosophy and the Making of Modernity 1650-1750 (traduit sous le titre Les Lumières Radicales), un excellent spécialiste de l'histoire économique des Pays-Bas, nommé Jonathan I. Israel, propose d'établir une sorte de cadastre de cette période en concevant la vie intellectuelle comme structurée par des fronts. Il oppose en particulier un courant modéré et un autre, « radical», formé autour de Spinoza, comme des mouvements intellectuels dessinant des propositions cohérentes. En particulier, le front « radical» se structure autour d'une série de positions : «Radical Enlightenment is a set of basic principles that can be summed up concisely as: democracy; racial and sexual equality; individual liberty of lifestyle; full freedom of thought, expression, and the press; eradication of religious authority from the legislative process and education; and full separation of church and state ${ }^{\mathrm{ix}} »$. Ayant déterminé ces « principes », J. Israel les emploie comme une sorte de questionnaire qui permet de tester l'appartenance d'un auteur à ce mouvement. A partir de questions fermées (est-ce que l'auteur adhère oui ou non à telle ou telle position ?), il peut ranger les personnages historique dans un camp ou dans l'autre. Bien sûr, presque personne ne remplit tous les critères qui définissent la radicalité, mais cette méthode permet de dessiner les contours du mouvement d'une manière pour ainsi dire empirique. Ce qui avait l'aspect du désordre généralisé devient quelque chose qui a du sens : il y a des courants d'idées dont les rapports déterminent le sens de l'histoire.

Cette méthode a ceci d'intéressant qu'elle permet de créer un objet que Jonathan Israel appelle le " spinozisme », puis de le suivre à travers les siècles et de l'identifier malgré ses mutations. Mais elle a trois principaux inconvénients, liés à sa (trop ?) vaste focale :

1. Elle attribue un projet commun à des acteurs qui ne l'admettraient pas comme tel. Par exemple, Spinoza est bien un démocrate et il conçoit son œuvre dans le cadre d'un projet collectif, mais aucun membre de ce collectif ne comprend le projet de la même manière. La méthode d'Israel bute donc sur la « communauté » d'idées qu'elle est censée déceler. Elle attribue aux auteurs, comme par capillarité avec le groupe, des idées qui ne sont pas les leurs.

2. Elle atténue les nuances et les ambiguïtés de chaque pensée. Par la simplicité anhistorique des questions qu'elle leur pose, elle fabrique des auteurs qui défendent de manière polémique des choses simples alors que, pour une part importante d'entre eux, ils écrivent des textes subtils qui tentent souvent de déjouer les conflits plus souvent que d'y prendre position.

3. Elle méconnaît le caractère contradictoire de certaines positions, ou leur rôle historique ambigü. Pour prendre un cas qu'il n'envisage pas, des femmes philosophes comme 
Anna Maria van Schurman (1607 - 1678) ou Anne Conway (1631 - 1679) témoignent d'une évolution en faveur des femmes, mais elles défendent des idées très conservatrices!

Or, l'une des raisons pour lesquelles Jonathan Israel procède à ce tri, c'est qu'il désire suivre le mouvement qu'il appelle le «spinozisme » depuis sa naissance à Amsterdam jusqu'à nos jours (ce qui a fait l'objet de quatre livres successifs). En définitive, le plus intéressant de son projet tient donc en ceci, qu'il montre que la volonté de clarifier une situation historique est liée à celle d'adapter les «leçons» qu'on pourrait en tirer (les fameuses « leçons de l'histoire ») à nos contemporains. En ce sens, l'effort de clarifier en histoire ne procède pas d'une nécessité intellectuelle ou d'une volonté de comprendre, mais d'un effort pour pratiquer les disciplines historiques afin d'éclairer sa propre époque. Clarifier le désordre n'est pas fait pour comprendre l'histoire, mais pour l'utiliser. Ajoutons qu'il n'y a pas de raison de rejeter ce projet, à condition de l'embrasser en toute conscience. Or, l'inconvénient de cette simplification est qu'elle permet de voir un peu trop clair dans une situation plus intriquée qu'on ne le voudrait. Il n'est pas interdit de mettre en suspend cet objectif politique (la nécessité de " faire quelque chose » ou de rendre le récit d'un événement "signifiant» pour le monde contemporain) et tâcher de regarder la situation historique de plus près.

\section{Vers la complexité.}

Rien n'indique qu'en acceptant le désordre en histoire, on se condamnerait à la rendre inintelligible, car cela relève d'une conception trop rigide de l'ordre. Sous le chaos des événéments, sous la diversité des auteurs, sous le brouhaha des échanges intellectuels, nous n'avons pas besoin de postuler l'existence de chaînes causales linéaires, même intriquées. En tenant mieux compte du rôle de l'observateur dans le travail historien, nous devons accepter que tout(e) historien(ne) façonne ses objets à partir des limites qu'il ou elle lui attribue ; et que c'est précisément par la manière dont on travaille les documents et interagit avec eux que l'on peut retrouver, mais d'une manière complexe et circulaire, la notion de vérité.

Si l'on accepte d'intégrer le désordre, alors il devient possible de faire admettre aux historiens des idées et même aux philosophes une évidence qu'ils exploitent peu dans leurs travaux: c'est que la vie quotidienne, les manières de manger, de faire ses courses, de se déplacer, d'aller à l'église, de faire des enfants, etc., ne sont pas indifférentes aux disciplines abstraites. Bien sûr, le repas du déjeuner ne détermine pas de manière claire la résolution d'une équation mathématique dans l'aprèsmidi. Dans les disciplines de l'abstraction, le lien entre les événements de la vie quotidienne et l'évolution des réponses techniques à des problèmes techniques n'est ni perceptible, ni immédiat. Mais les études sur l'histoire des mœurs ou les pratiques sociales n'en sont pas moins indispensables pour décloisonner ce qu'on appelle l'histoire de la philosophie. Alors, comment s'y prendre ?
Entre l'alimentation et les innovations en métaphysique ou en mathématique, le hiatus ne vient pas de l'hétérogénéité des domaines, il vient de la linéarité de la consécution causale. Les démonstrations les plus abstraites sont évidemment dépendantes des livres que l'on a lus mais aussi des conditions d'accès aux livres et aux bibliothèques, des personnes que l'on fréquente ou que l'on ne doit pas fréquenter, de celles envers qui l'on veut se montrer fidèle pour des raisons familiales et affectives, etc. Il n'y a donc aucune contre-indication à mobiliser les dimensions les plus triviales de l'existence humaine pour éclairer la philosophie, du moins tant que l'on ne conçoit pas le lien entre les domaines comme une consécution causale linéaire.

Quelle conceptualité faut-il donc se donner pour respecter le désordre en histoire ? Une piste consiste à concevoir l'histoire elle-même comme un travail d'abstraction. Ce travail a pour but de passer d'un désordre objectif (les embarras de l'existence) à un chaos considéré de manière formalisée, dont le récit est une première étape. Par exemple, au lieu de se demander d'où vient le concept de substance chez Spinoza, quels sont les auteurs qui l'ont « influencé » et comment mesurer la différence entre eux, on peut choisir de laisser le désordre tel qu'il est : à savoir, d'un côté, des conceptions néothomistes, des conceptions cartésiennes, mais aussi des pratiques de lecture religieuse, les éditions successives des Eléments d'Euclide, etc. Bien entendu, la mathématique ou les réunions des collégiants qui commentent le Nouveau Testament n'ont rien à voir avec la métaphysique, mais c'est précisément ici que le désordre devient descriptible : il est possible d'établir un traçage des transferts conceptuels d'un domaine à l'autre et de montrer comment des rencontres improbables engendrent des effets inattendus. Si par exemple vous faites réagir la notion cartésienne d'infini positif avec l'usage du microscope en anatomie, il s'ensuit des effets qui se donnent à lire sous la plume de Spinoza. Une idée est transformée par une autre qui n'a rien à voir, afin de résoudre un problème dans un troisième domaine (comme je l'ai fait ailleurs, je n'y reviens pas ici ${ }^{\mathrm{x}}$ ).

Or, ce dispositif n'a plus rien de linéaire. Il ne s'agit pas de chaînes d'auteurs qui se suivent les uns après les autres dans un dialogue anhistorique, et qui propose des solutions (on ne sait d'où venues) à des problèmes (que seuls les historiens formulent) mais plutôt de références hétérogènes qui interfèrent, à un point précis identifiable de l'histoire intellectuelle (en l'occurrence, «chez Spinoza ») et qui deviennent ce qu'on peut appeler des « inter-références ». Au reste, le nombre de références est indifférent, car l'un des privilèges de cette méthode est qu'elle ne prétend pas épuiser la complexité historique. A vrai dire, seul l'historien lui-même définit, par les limites de son érudition, l'extension de son désordre de référence. Sur ces bases, il n'est plus nécessaire de trier entre les camps philosophiques, puisqu'on étudie les interactions plus que les auteurs. Il n'est plus besoin de simplifier les fronts, car on explore des mouvements qui sont ambivalents, où les adversaires apparaissent à égalité (ce qui laisse les préférences s'exprimer autrement). Dans ces conditions, l'histoire intellectuelle s'assume comme une 
pratique expérimentale et exploratoire, et l'on peut émanciper le désordre de tout effort de clarification.

i Lettre 32 de Spinoza à Oldenburg, in B. de Spinoza, Correspondance, ed. et trad. M. Rovere, Paris, Garnier Flammarion (2010), p. 212

ii B. de Spinoza, Ethique, trad. C. Appuhn, Paris, Bouquins (2019), p. 632

iii Lettre 33 de Oldenburg à Spinoza, in Correspondance, op. cit., p. 213

iv Lettre 12 de Spinoza à Meyer, in Correspondance, op. cit., p. 98

v Id., ibid., p. 100

vi Id., ibid., loc. cit.

vii Spinoza, Ethique, part. II, scolie du lemme 7 après la proposition 13, scolie, op. cit., p. 652 viii Une exception à cette attitude est celle de B. Lahire, selon qui « « le social gît dans les détails et dans le singulier», tandis que le plus souvent, «le chercheur concentre ses efforts sur l'étude des acteurs et des institutions et arrive épuisé devant les portes d'un « palais textuel» dont il ne décrit finalement l'architecture qu'à gros traits » (Bernard Lahire, Franz Kafka, Éléments pour une théorie de la création littéraire, Paris, La Découverte, 2010, p. 33)

ix J. I. Israel, Radical Enlightenment: Philosophy and the Making of Modernity, 1650-1750, Oxford University Press (2001)

x Voir principalement M. Rovere, Le clan Spinoza, Paris, Flammarion (2017) 